\title{
A SOLUTION TO THE OVER-PRODUCTION OF TURBULENCE BENEATH SURFACE WAVES IN RANS MODELS
}

David R. Fuhrman, Technical University of Denmark, drf@mek.dtu.dk

Bjarke Eltard Larsen, Technical University of Denmark, bjelt@mek.dtu.dk

\section{BACKGROUND AND INTRODUCTION}

CFD simulations of breaking waves with RANS models over the past 20 years have shown a marked tendency to severely over-predict turbulence levels, both outside and within the surf zone. The reason is most likely due to the inherent instability of turbulence closure models, as originally diagnosed by Mayer \& Madsen (2000). While they proposed an ad-hoc fix, a fundamentally sound solution to this wide-spread problem has, to date, eluded the coastal and ocean engineering modeling community.

Mayer \& Madsen (2000) showed that the potential flow region beneath surface waves gives rise to non-zero turbulence production, and that this can cause standard two-equation closures to be conditionally unstable. In this work we analyze several widely-used RANS turbulence closure models (both k- $\omega$ and k- $\varepsilon$ type). We prove that all are, in fact, unconditionally unstable, inevitably resulting in exponential growth of both the turbulent kinetic energy and eddy viscosity. The respective unstable growth rates for several models are newly derived in closed form.

We further prove that through simple and elegant modifications to their "stress-limiting" features, e.g. defining the eddy viscosity $v_{T}$ in the k- $\omega$ model as:

$$
v_{T}=\frac{k}{\widetilde{\omega}}, \quad \widetilde{\omega}=\max \left\{1, \lambda \frac{S_{i j} S_{i j}}{\Omega_{i j} \Omega_{i j}}\right\} \omega
$$

while also eliminating $v_{T}$ from the $\omega$ production term, that standard closures can be formally stabilized (similarly for k- $\varepsilon$ models). In (1) $S_{i j}$ and $\Omega_{i j}$ are, respectively, the mean strain- and rotation-rate tensors, and $\lambda \ll 1$ is a new stress-limiter coefficient. This solution allows the closure model to naturally default to a standard form in shearedflow regions e.g. the bottom boundary layer and surf zone, while formally curing the instability in the potential flow region, thus solving this long-standing problem.

\section{SIMULATION OF A SIMPLE STREAM FUNCTION WAVE}

To most clearly demonstrate the performance of a standard versus new stabilized closure, a simple stream function wave $(\mathrm{kh}=0.66$ and $\mathrm{kH}=0.21)$ is first simulated in a CFD model. To mimic potential flow as closely as possible a slip bottom boundary condition is used. Hence, $v_{T}$ should ideally remain negligible and the wave should maintain its form. Fig. 1 shows the temporal development of $v_{T} / v$ for the standard and new stabilized closure models. As predicted, the standard closure exhibits exponential growth of the eddy viscosity. This ultimately becomes several orders of magnitude larger than the kinematic viscosity, leading to unphysical decay of the wave (Fig. 2a). This canonical test involving the long-term steady propagation of a surface wave train is one that standard (yet widely-used) RANS closure models simply cannot pass! Conversely, the new modified stabilized closure exhibits desirable decay of $v_{T}$, and the wave maintains nearly constant form (Fig. 2b).

Significantly improved results using the new stabilized closure will also be presented from more advanced CFD simulations of breaking waves. It will be shown that the new stabilized closure eliminates entirely the wide-spread problem of over-predicted pre-breaking turbulence, with corresponding major qualitative improvements in the preto-post breaking evolution of the undertow profiles. Further details are provided in Larsen \& Fuhrman (2018).

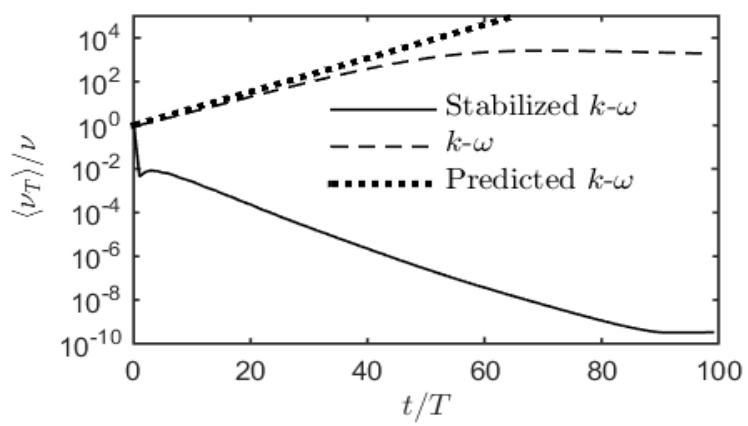

Figure 1 - Temporal development of the period- and depthaveraged eddy viscosity for two different turbulence models.
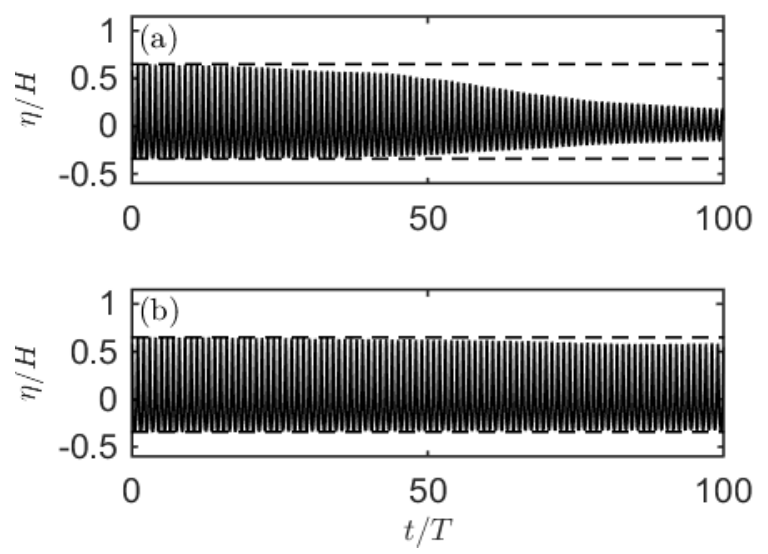

Figure 2 - Surface elevations using (a) the standard k- $\omega$ model and (b) the new stabilized k- $\omega$ model.

\section{REFERENCES}

Larsen, B.E. \& Fuhrman, D.R. (2018) On the overproduction of turbulence beneath surface waves in RANS models. J. Fluid Mech. (in revision).

Mayer, S. \& Madsen, P.A. (2000). Simulation of breaking waves in the surf zone using a Navier-Stokes solver. In: Proc. 25th ICCE. Sydney, Australia, pp. 928-941. 